Théologiques

Théologiques

\title{
L'hésychasme, paix du coeur au coeur du monde : pour ne pas s'enliser
}

\section{Horia Roscanu}

Volume 7, numéro 2, automne 1999

Silence !

URI : https://id.erudit.org/iderudit/005026ar

DOI : https://doi.org/10.7202/005026ar

Aller au sommaire du numéro

\section{Éditeur(s)}

Faculté de théologie de l'Université de Montréal

\section{ISSN}

1188-7109 (imprimé)

1492-1413 (numérique)

Découvrir la revue

\section{Citer cet article}

Roscanu, H. (1999). L'hésychasme, paix du coeur au coeur du monde : pour ne pas s'enliser. Théologiques, 7(2), 95-103. https://doi.org/10.7202/005026ar
Résumé de l'article

Le silence dans la cité est souvent fort difficile à réaliser. La spiritualité desÉglises d'Orient propose un rapport au réel qui cherche à faire une place àce silence au coeur du quotidien, à un recentrement sur l'essentiel qui n'estpas fuite au dehors mais vie au dedans. 


\title{
L'hésychasme, paix du cœur au cœur du monde : pour ne pas s'enliser
}

\author{
H oria ROSCANU \\ Candidat au Ph.D. en théologie \\ Université de M ontréal
}

\section{U ne théologie hors les murs}

À partir du commandement évangélique « Tu aimeras le Seigneur, ton Dieu, de tout ton cœur, de toute ton âme, de toute ta force (ou volonté) et de tout ton esprit ; et ton prochain comme toi-même » ( $L$ c 10, 25-27), Williams et $M$ cK ibben ${ }^{1}$ déduisent trois sortes de théologies, qui sont complémentaires, et qui doivent aller de paire dans une vie spirituelle équilibrée:

1) une théologie centrée sur l'âme (foi) : pensée

2) une théologie centrée sur le cœur (espérance) : sentiment

3) une théologie centrée sur la volonté (charité) : action

Ces approches ont malheureusement été éclipsées par l'une des trois, la théologie faisant appel à la pensée, et c'est la seule qui est formellement enseignée dans nos systèmes d'enseignement actuels. Les chrétiens de toutes traditions en sont victimes à des degrés divers.

Sans un équilibre dynamique entre les trois sortes de théologie, la nature humaine déchue a proclamé la théologie scolaire meilleure que les deux autres. II n'est dès lors pas étonnant que les chrétiens ayant peu d'enclin pour la théologie scolaire se sentent comme des citoyens de deuxième Classe dans l'Église ; ils ont été dorlotés dans ce genre de sentiments. ${ }^{2}$

Une authentique et saine théologie a besoin des trois aspects. En effet, sans une base théologique formée de cœur et de volonté, la théo-

1. Benjamin D. WILLIAM S et M ichael T. M CKIBBEN, O riented L eadership, Wayne (N J), O rthodox Christian Publication Center, 1994, p. 37-40.

2. Ibid., p. 39. 
logie centrée sur la pensée s'assèche dans le rationalisme et se désincarne ; elle devient système de théorêmes célestes, vue del'esprit, «pelletage de nuages » sans prise sur la réalité. De même, sans une base théologique formée de volonté et de pensée, la théologie centrée sur le cœur et le sentiment devient vaine, orgueilleuse, et envieuse, elle ira au gré des émotions et des modes, telle une plante anémophile, sans ancrage d'intelligence historique. Enfin, sans une base théologique formée de cœur et de pensée, une théologie centrée sur la volonté devient paresseuse, lascive, et colérique. ${ }^{3}$

Avec la méthode hésychaste, la tradition chrétienne orientale vient remettre l'équilibre entre ces trois pans complémentaires de la théologie, et combler les attentes des chrétiens qui cherchent un complément indispensable de vie spirituelle à leurs recherches intellectuelles. Elle affirme avec force que « si tu es théologien, tu prieras vraiment, et si tu pries vraiment, tu es théologien ». ${ }^{4}$ D ès lors, la théologie entendue comme connaissance intime de Dieu n'est plus le monopole des acadé miciens et des clercs instruits, elle est à la portée de tous, elle dépasse de loin les frontières de l'Université, elle en est quelquefois même exilée...

$M$ ais qu'est-ce que la méditation hésychaste? Le mot grec hsuxia (hésychia) signifie «calme, paix, sérénité, silence, recueillement, quiétude, la marque d'un intérieur unifié $» .{ }^{5}$ Pour résumer lapidairement, on peut dire que l'hésychasme est la prise de conscience que «le R oyaume de Dieu est au-dedans de vous» (LC 17,21). La méthode de prière, centrée sur l'invocation du N om de Dieu, et plus particulièrement le N om de Jésus, la posture corporelle, le chapelet de laine, ne sont que des supports pour chercher à « libérer le dynamisme de l'Esprit enfoui dans le cœur humain $» .{ }^{6}$ L'essentiel est de " demeurer devant Dieu, avec l'intellect dans le coeur, et de continuer à se tenir ainsi devant lui, sans cesse, jour et nuit, jusqu'à la fin de sa

3. Ibid., p. 39-40.

4. Évagre le Pontique, dans Petite philocalie de la prière du cœur, traduite et présentée par J ean Go UILLARD, Paris, Seuil, 1979, p. 42.

5. Lucien CouTU, C.S.C., L a méditation hésychaste, M ontréal, Fides, 1996, p. 27. Cet ouvrage constituel'une des meilleures introductions à la spiritualité hésychaste. L'auteur donne régulièrement des sessions d'initiation au Centre Emmaüs qu'il a fondé il y a 28 ans, ou ailleurs, selon les demandes.

6. Lucien COUTU, La méditation hésychaste, p. 40. 
vie ${ }^{7}$ L'hésychasme représente le cœur intime de la spiritualité orthodoxe, le choix de « la meilleure part » (L c 10,42), la réponse au commandement « quand tu veux prier, entre dans ta chambre » (M t 6,6), la chambre du cœur, le centre unifiant de l'être. Le mot de l'A pôtre «Priez sans cesse » $(1$ Th 5,17) devient ainsi un but réaliste.

\section{Le monachisme intériorisé, ou « pas de spiritualité laïque »}

L'O ccident médiéval a précisé autrefois que le monachisme répond aux consilia de l'Évangile, le laïcat répondant aux prœcepta de celui-ci. A utrement dit, d'une part, les parfaits, les moines, de l'autre, les faibles, les laïcs vivant dans le clair-obscur hasardeux de la Cité terrestre. La vie conjugale n'est par conséquent tolérée qu'en tant qu'elle engendre des vierges et peuple les couvents! D'où il en est résulté le fâcheux clivage clerc / laïc, enseignants / enseignés, clergé actif et puissant / laïcat passif et obéissant, dont on a grand'peine à sortir aujourd'hui. D'où aussi la recherche pressante et légitime d'une « spiritualité laïque » adaptée au monde contemporain : « II n'y a qu'une sainteté [...] M ais on est présentement en recherche d'une intelligence et d'une pratique de cette sainteté qui conviennent proprement au laïc d'Église, au service laïc dans l'Église ${ }^{8}$

L'O rient a cependant un autre son de cloche : l'exigence del'Évangile s'adresse à chacun-e, quel que soit son état. ${ }^{9}$ " Q uand le Christ ordonne de suivre la voie étroite, il s'adresse à tous les humains. Le moine et le séculier doivent atteindre les mêmes hauteurs » (S. Jean Chrysostome). Une seule spiritualité pour tous, l'idéal étant le même pour tous, seuls les degrés varient. Ainsi, I'O rient chrétien n'a pas développé de "spiritualité laïque», de "spiritualité du troisième âge », de «spiritualité du tourisme », du couple, des enfants, etc. Le

7. Saint Théophane le Reclus (1815-1894), cité par Kallistos WARE, Le royaume intérieur, Paris, Cerf, 1996, p. 84.

8. Yves CongAR, Jalons pour une théologie du laïcat, Paris, Cerf, 1954, p. 585.

9. «II n'y a qu'un christianisme [...] il n'y a pas de spiritualité propre des laïcs [...] le moine n'est qu'un chrétien qui pousse jusqu'au bout les exigences de l'unique nécessaire sans la primauté duquel il n'y a pas de vie chrétienne digne de ce nom. » Yves Con GAR, op. cit., p. 559-560. 
moine est d'ailleurs un simple laïc ${ }^{10}$ qui pousse jusqu'au bout le radicalisme de l'Évangile. D'ailleurs, les apophtegmes des Pères décrivent des laïcs engagés dans le monde qui ont dépassé les moines en perfection ; un médecin d'Alexandrie est même considéré l'égal de saint A ntoine le Grand, Père des moines. ${ }^{11}$

II y a eu dans l'histoire deux solutions pour vivre l'Évangile: 1) se retirer du monde, fuir au désert, s'enfermer dans le cloître monastique. $M$ ais on comprend que ce genre de vie n'est pas fait pour tous. 2) Christianiser le monde sans en sortir, bâtir la citéchrétienne : ce fut le but des théocraties. $M$ ais leur échec fut évident, car on ne peut jamais imposer I'Évangile par la force, et prescrire sa grâce comme une Loi : I'Églisen'a pas à imposer ses lois de la même façon qu'un pouvoir politique ${ }^{12}$ :

Le message chrétien [...] n'est pas une loi à imposer, mais une aimantation à proposer. II n'appartient pas à l'Église de dicter les lois de l'État ou de les bloquer commeun quelconque " groupe de pression ». L'Église inspire et sanctifie, elle ne contraint pas ; ce sont les coeurs qu'elle tente de changer. M ême pour ses fils I'Église doit donc être une mère miséricordieuse et non un pouvoir juridique impersonnel. ${ }^{13}$

Le théologien laïc Paul Evdokimov ${ }^{14}$ propose une troisième solution, celle du monachisme intériorisé, qui répond à l'exigence évangélique d'être dans le monde sans en être: "Vous êtes dans ce monde, vous n'êtes pas du monde ». Cette solution n'est pas vraiment nouvelle, dans le sens qu'elle est inhérente à la tradition hésychaste qui propose l'idéal monastique à tous. Encore faut-il interpréter, incarner cet idéal dans notre vie de laïcs engagés dans la cité terrestre.

10. À moins d'être ordonné, pour les besoins du monastère, au diaconat ou au presbytérat, ce qui n'est pas automatique.

11. Kallistos WARE, L e royaume intérieur, p. 86.

12. Voir H. ROSCANU, "'Une aimantation à proposer' : jalons pour une éthique sociale orthodoxe», Église et théologie, 27 (1996), 253-274.

13. Constantinos CHARALAM BIDIS, « Le mariage dans l'Égl ise orthodoxe», Contacts, 101 (1978), p. 70. D'où la réticence éprouvée par plusieurs chrétiens orientaux, en principe opposés à l'avortement, à s'associer à des groupes militants du genre pro-vie : ils savent quel'amour ne s'impose pas par des lois. 14. Paul Evdokimov, Les âges de la vie spirituelle, Paris, DDB, 1964, p. 121-146. 
Saint J ean Chrysostome donne le ton : « Ceux qui vivent dans le monde bien que mariés, doivent par tout le reste ressembler aux moines. Vous vous trompez tout à fait si vous pensez qu'il est des choses exigées des séculiers, et d'autres des moines. Ils auront les mêmes comptes à rendre $» .{ }^{15}$ CePère del'Égl ise veut-il dire que les laïcs doivent vivreles trois vœux monastiques au même titre que les moines? Pas vraiment, puisque l'Église n'a pas approuvé ceux qui affirmaient que la vie monastique est l'unique voie de salut. Prière, jeûne, lecture des Écritures, discipline ascétique sont ainsi proposés à tous au même titre, selon leurs capacités. Les jeûnes des Églises d'O rient sont prescrits à tous les fidèles mais peuvent être relâchés selon une certaine plasticité, au rythme de chacun et selon ses aptitudes. La norme est là, monastique et ascétique, les fidèles doivent y tendre de leur mieux, selon leurs possibilités, l'essentiel étant de grandir dans la vie spirituelle.

A insi, la pauvreté n'est pas forcément radicale, mais une libération de l'emprise du matériel, qui risque souvent de nous étouffer. C'est dans l'usage que l'on fait des biens matériels que réside la vraie pauvreté, c'est-à-dire le détachement versus l'étouffement dans les biens terrestres. La réussite matérielle comme critère de valeur est donc critiquée ici.

La chasteté est la libération de l'emprise du charnel. C'est voir en I'autre non un objet de plaisir, mais un visage, une personne, avec sa poésie et son mystère irréductible. II faut dire qu'en 0 ccident le terme chasteté est devenu peu à peu synonyme de non-génitalité, ce qui n'est pas le cas en Orient. Ainsi prie-t-on, dans la liturgie byzantine du mariage, pour que Dieu accorde aux époux « un amour chaste», c'est-à-dire plein de respect et de tendresse.

Chasteté veut aussi dire respect amoureux et non-exploiteur de la Création comme don du Très-H aut. C'est finalement le choix ultime entre le viol de la nature, la surconsommation boulimique, I'utilisation immodé rée des ressources de la planète, le capitalisme sauvage, l'usage de la force d'une part, et d'autre part l'action de grâces, l'eucharistia en toutes choses selon saint Paul, la chasteté pleine de tendresse à l'égard de la beauté dont nous sommes les gestionnaires responsables. C'est le renversement d'une autre valeur mondaine, celle du primat de l'efficacité sur la vérité.

15. Cité par P. Evdokimov, op. cit., p. 140. 
Quant au troisième vœu monastique d'obéissance, il s'agit de la libération de l'emprise idolâtre de l'égo. C'est être à l'écoute des appels de l'Esprit, obéir au Père qui l'envoie, répondre par l'affirmative et avec générosité à notre vocation filiale.

Evdokimov fait remarquer ${ }^{16}$ que le $\mathrm{N}$ otre $\mathrm{P}$ ère reprend les trois vœux : obéissance à la volonté du Père (que ta volonté soit faite) ; pauvreté : avoir faim du pain substantiel, eucharistique, unique nécessaire (donne-nous aujourd'hui notre pain de ce jour) ; chasteté : purification du M alin (délivre-nous du M al(in)).

\section{Au coeur du quotidien}

Aujourd'hui je vois la vie avec les yeux du cœur,J'suis plus sensible à l'invisible, à tout c'qu'il y a à l'intérieur...

Gerry Boulet

A u chevet de mon fils de cinq mois, à I'hôpital (une bronchiolite banale), par une nuit de décembre, me reviennent ces paroles de saint Silouane de I'A thos (1866-1938) : "Tiens ton esprit en enfer et ne désespère pas. » Comment garder les yeux du coeur fixés sur l'essentiel, comme laïc, père de famille, happé par les soucis réels et très prenants du quotidien? «Comment, si c'est la condition propre du laïc, être pleinement citoyen de la Cité à venir, non pas malgré l'engagement dans la Cité terrestre, mais dans cet engagement même? »17 Comment vivre le commandement «Priez sans cesse » $(1 \mathrm{Th} 5,17)$, qui nous est adressé à tous, sans exception, au cœur du monde?

Une nouvelle de Ion Agârbiceanu, prêtre et romancier roumain, raconte l'histoire d'un curé de village du XIX ${ }^{\mathrm{e}}$ siècle qui écrit une lettre à son évêque, au milieu de la nuit, pour lui demander de bien vouloir le remplacer temporairement dans ses tâches pastorales. Le pays connaissant la famine, et ce prêtre ayant une famille à nourrir et unefilleà marier, il désires'exiler pour les États-Unis, pour un ou deux ans, pays où il trouvera du travail et reviendra avec des économies. II pourra alors marier sa fille et reprendre ses fonctions pastorales avec plus d'entrain. Tout à coup, quelqu'un frappe à la porte: un villageois est sur le point de

16. Ibid.

17. Yves CONGAR, op. cit., p. 590. 
mourir ; son fils vient chercher le prêtre pour les derniers sacrements. Le prêtre se ressaisit, jette sa lettre dans le feu du foyer, enfile son manteau, prend son étole et le viatique, et s'enfonce dans la nuit...

Cette très belle histoire nous introduit à ce qui pourrait être un hésychasme dans le monde : il s'agit d'un esprit de veille intérieure, de centrage constant sur l'essentiel, en plein milieu des soucis quotidiens les plus terre-à-terre, une renonciation aux tentations de facilité de ce monde. H ésychasme au quotidien : essayer de ne pas perdre de vue le R oyaume qui vient, et que nous vivons déjà ici-bas, et qui affleure dans le sourire d'un enfant, dans le printemps qui surgit... Trouver Dieu au cœur du brouhaha quotidien, au milieu des courses, des couches à changer, des réunions, des biberons et des bains à donner, des articles à écrire, des coliques à calmer, des va-etviens à la garderie, des nuits interrompues où il semble parfois que les bébés veulent faire de leurs parents des moines A cémètes! ${ }^{18}$ Bien sûr, les tentations de fuite ne manquent pas, mais la prière du cœur, tel un silence profond qui transcende le tumulte de la vie, m'inscrit à nouveau au cœur du vécu, pour le transfigurer, pour y déceler la ténébreuse lumière de Dieu.

Prendre le temps de regarder, émerveillé, les couchers de soleil, les fleurs, les enfants, les mille et une beautés du quotidien, se remplir l'œil, sentir les parfums de la création et de la vie, faire prier ses yeux et son nez. N os oreilles accueillent les sons de la vie, le bruissement du vent dans les feuilles, le crissement de la neige sous nos raquettes, le cliquetis des branches givrées, le chant des hirondelles, le clapotis du ruisseau qui coule sur les roches, le roulement des vagues de la mer sur la plage. ${ }^{19}$

Pour que ce recentrage constant ait lieu, il est essentiel de se ressourcer périodiquement dans de belles liturgies hebdomadaires où le ciel effleure la terre, où les corps expriment la louange des cœurs par tous les sens ${ }^{20}$, pour briser la routine et sanctifier le quotidien. Le

18. M oines de Constantinople qui se levaient à chaque heure du jour et de la nuit pour un court office.

19. Lucien CoUTu, La méditation hésychaste, p. 32.

20. Les liturgies chrétiennes orientales sollicitent tous les sens: la vue (icônes, cierges), l'ouïe (chants), l'odorat (encens), le toucher (vénération des icônes), le goûter (communion sous les deux espèces). L'absence de bancs favorise la prière de l'être tout entier. 
chrétien en effet n'est pas seul, mais membre d'une communauté de croyants qui célèbre le salut et la victoire du Christ sur la mort et les enfers. Ainsi est étanchée périodiquement la soif de beauté et de communion. L'icône, fenêtre sur le R oyaume, est une bonne aide domestique pour se recentrer sur l'essentiel, un rappel quotidien de notre vocation de porteurs de lumière dans le monde, un appel à transfigurer le cosmos tout entier.

\section{Conclusion}

II nous faut des hommes de silence, nourris d'étonnement, d'attention, de « prière pure » et de beauté liturgique, pour dire une parole libératrice. ${ }^{21}$

M on fils de quatre ans Émilien ferme les yeux quand il caresse les oreilles de $\mathrm{N}$ icolas, son petit frère de huit mois, et arbore un sourire ludique. Q uel adulte peut prétendre posséder une telle attention, une telle concentration dans ses tâches quotidiennes? La méditation hésychaste enseigne à recentrer son esprit, à le diriger vers le centre, vers I'essentiel. Elle acquiert ainsi, sans vraiment y prétendre, une pertinence pour notre monde moderne : capacité de concentration, vivre le moment présent, calme, paix intérieure, autant de qualités de plus en plus recherchées chez les hommes et femmes d'affaires, les gestionnaires, les athlètes, etc. En fait, il semble se dessiner, en cette aube de siècle spirituel, un réel besoin d'hommes et de femmes qui tendent à l'unification de leur être, des personnes qui ont intégré leurs valeurs spirituelles, et qui démontrent par leur vécu qu'ils et el les prennent soin de toutes les facettes de leur personne, physique, psychique, émotive, spirituelle. Dans le monde des affaires, l'heure n'est plus à la honte d'affirmer ses valeurs, sa foi, sa spiritualité, comme ce fut le cas dans les années '80. Les entreprises vraiment modernes, celles qui survivront au tournant du siècle, sauront respecter les personnes qu'elles emploient, dans toutes leurs facettes. Elles ne leur demanderont plus, comme par le passé, de laisser leur spiritualité au vestiaire, de n'être que des fourmis productives et rationnelles de la fable de $L a$ Fontaine; elles feront aussi place à la cigale qui chante les merveilles du Seigneur au plus profond de nous, sachant libérer les

21. Olivier CLÉMENT, Corps de mort et de gloire, P?aris, DDB, 1995, 
potentialités spirituelles pour le mieux-être des personnes, une « écologie du management ». Preuve en est le récent Forum international sur le management, l'éthique et la spiritualité, qui s'est tenu à l'école des $\mathrm{H}$ autes Études $\mathrm{C}$ ommerciales de $\mathrm{M}$ ontréal. ${ }^{22}$

L'hésychasme conduit au silence intérieur, et ce silence va de pair avec la théologie apophatique, cette approche négative du $M$ ystère développée par les Pères grecs, pour le préserver de toute rationalisation comme de tout anthropomorphisme: « en procédant par négations, on s'élève à partir des degrés inférieurs de l'être jusqu'à ses sommets, en écartant progressivement tout ce qui peut être connu, afin de s'approcher de l'Inconnu dans les ténèbres de l'Ignorance absolue $» .{ }^{23} \mathrm{~A}$ insi comprend-on que Dieu est au-delà de tout, dans la «nuée obscure » (Ex 20,21), de même que certaines expériences humaines sont au-delà de tout langage.

\begin{abstract}
RÉSUMÉ
Le silence dans la cité est souvent fort difficile à réaliser. La spiritualité des Églises d'O rient propose un rapport au réel qui cherche à faire une place à ce silence au cœur du quotidien, à un recentrement sur l'essentiel qui n'est pas fuite au dehors mais vie au dedans.

\section{ABSTRACT}

Silence in the city is difficult to achieve. The spirituality of the Eastern Churches proposes to establish a relation with reality, a relation which tries to find a place for this silence in the midst of daily life by refocussing on the essential. It is not an escape from without but a living from within.
\end{abstract}

22. Premier Forum international sur le management, l'éthique et la spiritualité (FIM ES), École des H .É.C., M ontréal, 18-19 septembre 1998. Voir à ce sujet Thierry PAUCH ANT et collaborateurs, La Q uête du sens. G érer nos organisations pour la santé des personnes, de nos sociétés et de la nature, M ontréal, Q uébec/A mérique, 1996.

23. Vladimir L OSSKY, Essai sur la théologie mystique de l'É glise d'O rient, Paris, Cerf, 1990, p. 23. 